JOURNAL OF

FUNCTION SPACES AND APPLICATIONS

Volume 4, Number 2 (2006), 145-161
(C) 2006, Scientific Horizon http://www.jfsa.net

\title{
On the uniform exponential stability of linear skew-product semiflows*
}

\section{Ciprian Preda}

\author{
(Communicated by Björn Birnir)
}

2000 Mathematics Subject Classification. 34D05, 47D06, 93D20.

Keywords and phrases. Evolutionary processes, exponential stability, Orlicz spaces, admissibility.

\footnotetext{
Abstract. The problem of uniform exponential stability of linear skew-product semiflows on locally compact metric space with Banach fibers, is discussed. It is established a connection between the uniform exponential stability of linear skewproduct semiflows and some admissibility-type condition. This approach is based on the method of "test functions", using a very large class of function spaces, the so-called Orlicz spaces.
}

\section{Introduction}

It is known that many equations from physics can be cast as an abstract Cauchy problem

$$
\text { (A) } \quad x^{\prime}(t)=A(t) x(t), x(s)=x_{s} \in D(A(s)), \quad t \geq s, \quad t, s \in J,
$$

${ }^{*}$ This research was done while the author was a PostDoctoral Fellow at the Dept. of Electrical Engineering of UCLA, U.S.A. Research supported in part under NSF Grant No. ECS-0400730. 
on a Banach space $X$, where $J=\mathbb{R}$ or $J=\mathbb{R}_{+}$. Some systems can be described by a Cauchy problem, as for instance the heat equation, the Schrödinger equation, or certain population equations. Also, even the Maxwell's equations can be transformed into a Cauchy problem with slightly more effort. But also seemingly unrelated problems such as delay equations, Markov processes or Boltzmann's equations can be interpreted as Cauchy problems by choosing the "right" state space. Suppose now that the Cauchy problem $(A)$ is well-posed, which means that there exists an evolution family of operators $\{U(t, s)\}_{t \geq s}$, which solves the problem above $\left(x(t)=U(t, s) x_{s}\right)$. If $\{A(t)\}_{t \geq 0}$ is a bounded linear operators family which belongs to $M^{1}$ (i.e. $\left.\sup _{t \geq 0} \int_{t}^{t+1}\|A(\tau)\| d \tau<\infty\right)$, then the problem (A) is well-posed and generate a reversible evolution family.

If $f$ is a $X$-valued locally integrable function on $I$ we will consider the inhomogeneous equation:

$$
x^{\prime}(t)=A(t) x(t)+f(t), \quad t \in I .
$$

In this context we refer to a function $u(\cdot)$ as a mild solution of $(\mathrm{A}, \mathrm{f})$ if it is the solution of the inhomogeneous mild equation

$$
u(t)=U(t, s) u(s)+\int_{s}^{t} U(t, \tau) f(\tau) d \tau, \quad t \geq s, \quad t, s \in J .
$$

The connection between the uniform exponential stability of (A) and the solution of $(*)$ is originating in the famous paper of O. Perron [18] from 1930. This idea was extensively analyzed also in the monographs of Massera-Schäffer [16] and Daleckij-Krein [7].

An important extension of these above facts can be found when we are dealing with the theory of linear skew-product (semi)flows instead of the particular case of evolution families. As it is known the linear skew-product semiflows arise as solution operators for variational equation

$$
\frac{d}{d t} u(t)=A(\sigma(\theta, t)) u(t)
$$

where $\sigma$ is a semiflow on a locally compact space and $A(\theta)$ an unbounded linear operator on a Banach space $X$, for each $\theta \in \Theta$.

It is also relatively known that the qualitative behavior of (semi-)flows on (locally) compact spaces or $(\sigma$-)finite measure spaces is pointed out by concepts as stability or exponential dichotomy of the associated linear skewproduct (semi-)flow. The theory of linear skew-product flows with finite dimensional fibers has come in our days into widespread usage in the area in asymptotics theory of differential equations (see e.g. [9, 10, 22, 23, 26]). 
Also, it is well known the approach of J. Hale (10) which stressed that this theory should be extended in the more general framework of the infinite dimensional setting. In fact, in the last decade many contributions were done in the study of linear skew-product flows with Banach fibers over a compact metric space $[1,2,3]$. Also, many applications were done in $[2,9$, $10,11,25]$.

In the finite dimensional context the Sacker-Sell spectrum offered an interesting way to describe all of these properties (see $[9,23,24,25])$. This kind of approach was extended in the last decade to the case of normcontinuous cocycles on infinite dimensional Banach spaces by Latushkin and Stepin. Also, it is interesting to see that almost all interesting infinite dimensional situations, as for instance flows originating from partial differential equations and functional differential equations, only yield strongly continuous cocycles. In this context, there has been studied the dichotomy of linear skew-product semiflows defined on compact spaces (see $[2,3,4,5]$ ), and on a locally compact spaces, respectively (see [15]). The idea of associating an evolution semigroup in the expanded case of exponential stability or dichotomy of linear skew-product flow on locally compact metric space $\Theta$, has its origins in the works of Latushkin and Stepin [13], respectively Latushkin, Montgomery-Smith and Schnaubelt [14].

We can notice in this context the idea of "autonomization" of nonautonomous problems by passing from the case of evolution families or more general of linear skew-product semiflows to associated evolution semigroups.

In contrast to this "philosophy", the present paper shows that we can characterize also the exponential stability in terms of the admissibility of some suitable function spaces in a direct way, without so-called evolution semigroups. But of course, this is not the first aim of our paper.

We are setting an connection between the uniform exponential stability of a linear skew-product semiflow on a locally compact metric space and the admissibility of a function space from a large class of function spaces, the so-called Orlicz spaces. Therefore, it is considered a concept of exponential stability for linear skew-product semiflows, which is an extension of the classical concept of exponential stability for time-dependent linear differential equations in Banach spaces (see e.g $[6,7,8,16,17]$ ).

Until now, the most common function spaces used for the connection between admissibility and stability were $L^{p}$-spaces, which are in particular Orlicz spaces, so with this approach it can be obtained also an unified treatment, generalizing the above results. 


\section{Preliminaries}

In the next we list the principal notations and symbols. For $X$ a Banach space we denote by $\mathcal{M}\left(\mathbb{R}_{+}, X\right)$ the space of all measurable functions (in the sense of Bochner) from $\mathbb{R}_{+}$to $X$ and by:

$$
\begin{aligned}
& L_{\text {loc }}^{1}\left(\mathbb{R}_{+}, X\right)=\left\{f \in \mathcal{M}\left(\mathbb{R}_{+}, X\right): \int_{K} \| f(t)\right) \| d t<\infty, \text { for each compact } \\
& \left.K \operatorname{in} \mathbb{R}_{+}\right\} ; \\
& L^{p}\left(\mathbb{R}_{+}, X\right)=\left\{f \in \mathcal{M}\left(\mathbb{R}_{+}, X\right): \int_{\mathbb{R}_{+}}\|f(t)\|^{p} d t<\infty\right\}, \text { where } p \in[1, \infty) ; \\
& L^{\infty}\left(\mathbb{R}_{+}, X\right)=\left\{f \in \mathcal{M}\left(\mathbb{R}_{+}, X\right): \text { ess } \sup _{t \in \mathbb{R}_{+}}\|f(t)\|<\infty\right\} \text { where, } p \in[1, \infty) .
\end{aligned}
$$

Also for $\Theta$ a locally compact metric space we denote by $C_{b}(\Theta, X)$ the space of all bounded continuous functions from $\Theta$ to $X$.

It is known that $L^{p}\left(\mathbb{R}_{+}, X\right), L^{\infty}\left(\mathbb{R}_{+}, X\right), C_{b}(\Theta, X)$ are Banach spaces endowed with the respectively norms:

$$
\begin{aligned}
\|f\|_{p} & =\left(\int_{\mathbb{R}_{+}}\|f(t)\|^{p} d t\right)^{\frac{1}{p}} \\
\|f\|_{\infty} & =\text { ess } \sup _{t \in \mathbb{R}_{+}}\|f(t)\| \\
\|f\|_{C_{b}(\Theta, X)} & =\sup _{\theta \in \Theta}\|f(\theta)\| .
\end{aligned}
$$

In order to simplify the notations we put $L^{p}:=L^{p}\left(\mathbb{R}_{+}, \mathbb{R}\right)$, for all $p \in$ $[1, \infty), L^{\infty}:=L^{\infty}\left(\mathbb{R}_{+}, \mathbb{R}\right), L_{l o c}^{1}:=L_{l o c}^{1}\left(\mathbb{R}_{+}, \mathbb{R}\right)$ and $\|f\|_{C_{b}(\Theta, X)}:=\|f\| \|$

Now, for more convenience, we recall the definition of Orlicz spaces.

Let $\psi: \mathbb{R}_{+} \rightarrow \mathbb{R}_{+}$be a non-decreasing function such that, $\psi(t)>0$, for all $t>0$. Define

$$
\Psi(t)=\int_{0}^{t} \psi(s) d s
$$

A function $\Psi$ of this form is called a Young function. For a measurable function $f: \mathbb{R}_{+} \rightarrow \mathbb{R}$ and the Young function $\Psi$, we define

$$
M^{\Psi}(f)=\int_{0}^{\infty} \Psi(|f(s)|) d s .
$$


The set $L^{\Psi}$ of all $f$ for which there exists $k>0$ that $M^{\Psi}(k f)<\infty$ is easily checked to be a linear space and endowed with the Luxemburg norm

$$
\|f\|_{\Psi}=\inf \left\{k>0: M^{\Psi}\left(\frac{1}{k} f\right) \leq 1\right\}
$$

the space $\left(L^{\Psi},\|\cdot\|_{\Psi}\right)$ becomes a Banach space.

Remark 2.1. It is easy to check that $\chi_{[0, t]} \in L^{\Psi}$ and $\left\|\chi_{[0, t]}\right\|_{\Psi}=\frac{1}{\Psi^{-1}\left(\frac{1}{t}\right)}$, for all $t>0$.

Example 2.1. The classical $L^{p}$-spaces are examples of Orlicz spaces.

The connection between Orlicz spaces and the $L^{p}$ spaces is given by

Remark 2.2. $L^{\Psi}=L^{p}$ if and only if $\Psi(t)=t^{p}$, for all $t \geq 0$.

The "only if" part is obvious. Conversely if $L^{\Psi}=L^{p}$ then we have that $\left\|\chi_{[0, t]}\right\|_{\Psi}=\left\|\chi_{[0, t]}\right\|_{p}$, for all $t>0$, and so $\Psi^{-1}(s)=s^{\frac{1}{p}}$, for all $s>0$, which implies that $\Psi(t)=t^{p}$, for all $t \geq 0$.

As it is known there are many other interesting examples of Orlicz spaces, but when it is involved an admissibility condition to prove the exponential stability of some differential system it is very convenient to use a small input space, in order to verify the admissibility condition for not so many "test" functions.

In order to find a "tiny" function space which can be used for instance as "input space", in our approach, we present bellow a function space which is contained in all $L^{p}$, for $p \in[1, \infty)$, and which is not a $L^{p}$-type space.

Example 2.2. If we take $\Psi(t)=e^{t}-1$ then $L^{\Psi} \subset L^{p}$, for all $p \in[1, \infty)$.

Indeed one can see that $t^{m} \leq m ! \Psi(t)$ for all $t \geq 0$ and all $m \in \mathbb{N}^{*}$ which implies that $L^{\Psi} \subset L^{m}$, for all $m \in \mathbb{N}^{*}$. Having in mind that $L^{m} \bigcap L^{m+1} \subset L^{p}$ for all $p \in[m, m+1]$, and all $m \in \mathbb{N}^{*}$, it follows that $L^{\Psi} \subset L^{p}$, for all $p \in[m, m+1]$, and all $m \in \mathbb{N}^{*}$.

In what follows, if $L^{\Psi}$ is a Orlicz space we denote by

$$
L^{\Psi}(X)=\left\{f \in \mathcal{M}\left(\mathbb{R}_{+}, X\right): t \mapsto\|f(t)\|: \mathbb{R}_{+} \rightarrow \mathbb{R}_{+} \text {belongs to } L^{\Psi}\right\} .
$$

Remark 2.3. $L^{\Psi}(X)$ is a Banach space endowed with the norm

$$
\|f\|_{L^{\Psi}(X)}=\|\| f(\cdot)\|\|_{\Psi} .
$$

Proof. First we can notice that if $f \in L^{\Psi}$ and $h \in \mathcal{M}\left(\mathbb{R}_{+}, \mathbb{R}\right)$ with $|h| \leq|f|$, then $h \in L^{\Psi}$ and $\|h\|_{\Psi} \leq\|f\|_{\Psi}$. 
Now, let us prove that if $u \in L^{\Psi}$ then $|u|<\infty$ a.e. Considering $A_{1}=\left\{t \in \mathbb{R}_{+}:|u(t)|=\infty\right\}$, it is easy to check that

$$
|u| \geq|u| \chi_{A_{1}} \geq \delta \chi_{A_{1}}, \quad \text { for all } \delta>0
$$

and so by that we noticed above, it follows that

$$
\chi_{A_{1}} \in L^{\Psi} \text { and }\left\|\chi_{A_{1}}\right\|_{\Psi}=0 .
$$

Now it is obvious that $m\left(A_{1}\right)=0$ and so $|u|<\infty$ a.e. In order to prove that $L^{\Psi}(X)$ is a Banach space, let $\left\{f_{n}\right\}_{n \in \mathbb{N}}$ be a Cauchy sequence in $L^{\Psi}(X)$. We will show that there exists $\left\{f_{n_{k}}\right\}_{k \in \mathbb{N}}$ a subsequence of $\left\{f_{n}\right\}$ and $f \in L^{\Psi}$ such that

$$
f_{n_{k}} \stackrel{L^{\Psi}(X)}{\longrightarrow} f, \quad f_{n_{k}} \rightarrow \text { f a.e. }
$$

Using the fact that $\left\{f_{n}\right\}_{n \in \mathbb{N}}$ is Cauchy sequence, it follows that there exists $\left\{f_{n_{k}}\right\}_{k \in \mathbb{N}}$ a subsequence of $\left\{f_{n}\right\}$ such that

$$
\left\|f_{n_{k+1}}-f_{n_{k}}\right\|_{L^{\Psi}(X)} \leq \frac{1}{2^{k}}, \quad \text { for all } \quad k \in \mathbb{N}
$$

It follows that the sum $\left(\sum_{k \geq 0}\left\|f_{n_{k+1}}-f_{n_{k}}\right\|\right)$ is absolute convergent in the Banach space $L^{\Psi}$. Let $g \stackrel{L^{\Psi}}{=} \sum_{k=0}^{\infty}\left\|f_{n_{k+1}}-f_{n_{k}}\right\|, g_{m}=\sum_{k=0}^{m}\left\|f_{n_{k+1}}-f_{n_{k}}\right\|$ and

$h: \mathbb{R}_{+} \rightarrow \overline{\mathbb{R}_{+}}, h(t)=\sum_{k=0}^{\infty}\left\|f_{n_{k+1}}(t)-f_{n_{k}}(t)\right\|$. It is easy to verify that

$$
g_{m} \stackrel{L^{\Psi}}{\longrightarrow} g, \quad \text { and } \quad g_{m}(t) \nearrow h(t), \text { for all } t \geq 0 .
$$

By the other hand

$$
\begin{aligned}
\int_{0}^{t}|g(s)-h(s)| d s & \leq \int_{0}^{t}\left|g(s)-g_{m}(s)\right| d s+\int_{0}^{t}\left|g_{m}(s)-h(s)\right| d s \\
& \leq t \Psi^{-1}\left(\frac{1}{t}\right)\left\|g_{m}-g\right\|_{L^{\Psi}}+\int_{0}^{t} h(s) d s-\int_{0}^{t} g_{m}(s) d s
\end{aligned}
$$

for all $t \geq 0$ and all $m \in \mathbb{N}$.

Using $(* *)$ we obtain that

$$
\int_{0}^{t}|g(s)-h(s)| d s=0, \quad \text { for all } \quad t \geq 0
$$


and hence $g=h$ a.e.

Let $A_{2}=\left\{t \in \mathbb{R}_{+}:|g(t)|=\infty\right\}, A_{3}=\left\{t \in \mathbb{R}_{+}: g(t) \neq h(t)\right\}, A_{4}=$ $A_{2} \bigcup A_{3}$. Knowing the fact that $g \in L^{\Psi}$ and $g=h$ a.e., we have that

$$
m\left(A_{4}\right)=0 \text { and } \sum_{k=0}^{\infty}\left\|f_{n_{k+1}}(t)-f_{n_{k}}(t)\right\|<\infty, \quad \text { for all } t \in \mathbb{R}_{+} \backslash A_{4},
$$

which implies that the subsequence $\left\{f_{n_{k}}(t)\right\}_{k \in \mathbb{N}}$ is convergent in $X$ for all $t \in \mathbb{R}_{+} \backslash A_{4}$.

Let $f: \mathbb{R}_{+} \rightarrow X$ the map given by,

$$
f(t)= \begin{cases}\lim _{k \rightarrow \infty} f_{n_{k}}(t), & t \in \mathbb{R}_{+} \backslash A_{4} \\ 0, & t \in A_{4}\end{cases}
$$

Thus we have that $f_{n_{k}} \rightarrow f$ a.e. and so $f$ is measurable and

$$
f(t)-f_{n_{m}}(t)=\sum_{k=m}^{\infty}\left(f_{n_{k+1}}(t)-f_{n_{k}}(t)\right),
$$

for all $t \in \mathbb{R}_{+} \backslash A_{4}$ and all $m \in \mathbb{N}$, therefore

$\left\|f(t)-f_{n_{m}}(t)\right\| \leq g(t)-g_{m-1}(t), \quad$ for all $\quad t \in \mathbb{R}_{+} \backslash A_{4} \quad$ and all $m \in \mathbb{N}^{*}$.

Thus, we have that $f-f_{n_{m}} \in L^{\Psi}$ for all $m \in \mathbb{N}^{*}$ and so $f \in L^{\Psi}$ and

$$
\left\|f-f_{n_{m}}\right\|_{L^{\Psi}(X)} \leq\left\|g-g_{m-1}\right\|_{\Psi}, \quad \text { for all } \quad m \in \mathbb{N}^{*} .
$$

It follows that $f_{n_{k}} \stackrel{L^{\Psi}(X)}{\longrightarrow} f, \quad f_{n_{k}} \rightarrow f$ a.e. Since $\left\{f_{n}\right\}_{n \in \mathbb{N}}$ is a Cauchy sequence with a convergent subsequence, then $\left\{f_{n}\right\}_{n \in \mathbb{N}}$ it is also convergent.

Remark 2.4. If $\left\{f_{n}\right\}_{n \in \mathbb{N}} \subset L^{\Psi}(X), f \in L^{\Psi}(X)$, and $f_{n} \rightarrow f$ in $L^{\Psi}(X)$ when $n \rightarrow \infty$, then there exists $\left\{f_{n_{k}}\right\}_{k \in \mathbb{N}}$ a subsequence of $\left\{f_{n}\right\}_{n \in \mathbb{N}}$ such that

$$
f_{n_{k}} \rightarrow f \text { a.e. }
$$

Proof. Since $f_{n} \stackrel{L^{\Psi}(X)}{\longrightarrow} f$ then $\left\{f_{n}\right\}_{n \in \mathbb{N}}$ is a Cauchy sequence and from the proof of the Remark 2.3. there exists $\left\{f_{n_{k}}\right\}_{k \in \mathbb{N}}$ a subsequence of $\left\{f_{n}\right\}$ and $g \in L \Psi$ such that

$$
f_{n_{k}} \stackrel{L^{\Psi}(X)}{\longrightarrow} g, \quad f_{n_{k}} \rightarrow g \text { a.e. }
$$

This shows that $f=g$ a.e. and so $f_{n_{k}} \rightarrow f$ a.e. 
Proposition 2.1. If $\Psi$ is an Young function of the Orlicz space $L^{\Psi}$ then

(i) The map $a_{\Psi}: \mathbb{R}_{+}^{*} \rightarrow \mathbb{R}_{+}^{*}$ given by $a_{\Psi}(t)=t \Psi^{-1}\left(\frac{1}{t}\right)$ is a nondecreasing one;

(ii) $\int_{0}^{t}|f(s)| d s \leq a_{\Psi}(t)\|f\|_{\Psi}$, for all $t>0, f \in L^{\Psi}$.

Proof. (i) First let us prove that the map $b: \mathbb{R}_{+}^{*} \rightarrow \mathbb{R}_{+}^{*}$, given by $b(u)=\frac{\Psi(u)}{u}$ is nondecreasing. If $0<u_{1} \leq u_{2}$ then

$$
\begin{aligned}
\frac{\Psi\left(u_{1}\right)}{u_{1}} & =\frac{1}{u_{1}} \int_{0}^{u_{1}} \psi(s) d s \\
& =\frac{1}{u_{1}} \int_{0}^{u_{2}} \psi\left(\frac{u_{1}}{u_{2}} v\right) \frac{u_{1}}{u_{2}} d v \\
& =\frac{1}{u_{2}} \int_{0}^{u_{2}} \psi\left(\frac{u_{1}}{u_{2}} v\right) d v \\
& \leq \frac{1}{u_{2}} \int_{0}^{u_{2}} \psi(v) d v \\
& =\frac{\Psi\left(u_{2}\right)}{u_{2}} .
\end{aligned}
$$

In order to prove that $a_{\Psi}$ is nondecreasing let $0<t_{1} \leq t_{2}$. It follows that $0<w_{2}:=\Psi^{-1}\left(\frac{1}{t_{2}}\right) \leq \Psi^{-1}\left(\frac{1}{t_{1}}\right):=w_{1}$ and so $b\left(w_{2}\right) \leq b\left(w_{1}\right)$. Having in mind that

$$
b\left(w_{1}\right)=\frac{1}{a_{\Psi}\left(t_{1}\right)} \quad \text { and } \quad b\left(w_{2}\right)=\frac{1}{a_{\Psi}\left(t_{2}\right)},
$$

it results that $a_{\Psi}$ is a nondecreasing function.

(ii) Consider $f \in L^{\Psi}, t>0, k>0$ such that $M^{\Psi}\left(\frac{1}{k} f\right) \leq 1$. Then we have that

$$
\Psi\left(\frac{1}{k t} \int_{0}^{t}|f(s)| d s\right) \leq \frac{1}{t} \int_{0}^{t} \Psi\left(\frac{1}{k}|f(s)|\right) d s \leq \frac{1}{t}
$$

and so

$$
\int_{0}^{t}|f(s)| d s \leq t \Psi^{-1}\left(\frac{1}{t}\right) k
$$

which implies that

$$
\int_{0}^{t}|f(s)| d s \leq t \Psi^{-1}\left(\frac{1}{t}\right)\|f\|_{\Psi}=a_{\Psi}(t)\|f\|_{\Psi},
$$

for all $t>0, f \in L^{\Psi}$. 
Remark 2.5. Using a simple translation argument we may state that

$$
\int_{t_{0}}^{t_{0}+t}|f(s)| d s \leq a_{\Psi}(t)\|f\|_{\Psi}
$$

for all $t_{0} \geq 0, t>0, f \in L^{\Psi}$.

Definition 2.1. A mapping $\sigma: \Theta \times \mathbb{R}_{+} \rightarrow \Theta$ is called a semi-flow on $\Theta$ if it has the following properties:

$\left(s f_{1}\right) \quad \sigma(\theta, 0)=\theta$ for all $\theta \in \Theta$;

$\left(s f_{2}\right) \quad \sigma(\theta, t+s)=\sigma(\sigma(\theta, s), t)$ for all $t, s \geq 0, \theta \in \Theta$;

$\left(s f_{3}\right) \quad \sigma$ is continuous.

In order to simplify the notations we will put $\sigma(\theta, t)=\theta \cdot t$

Definition 2.2. A pair $\pi=(\Phi, \sigma)$ is called a linear strongly continuous skew-product semi-flow on $\mathcal{E}=X \times \Theta$ if $\sigma$ is a semi-flow on $\Theta$ and $\Phi: \Theta \times \mathbb{R}_{+} \rightarrow B(X)$ satisfy the following conditions:

$\left(s p_{1}\right) \quad \Phi(\theta, 0)=I$ (the identity operator on $X$ );

$\left(s p_{2}\right) \quad \Phi(\theta, t+s)=\Phi(\sigma(\theta, t), s) \Phi(\theta, t)$ for all $t, s \geq 0, \theta \in \Theta$;

$\left(s p_{3}\right) \Phi$ is strongly continuous;

$\left(s p_{4}\right)$ there exists $M, \omega>0$ that

$$
\|\Phi(\theta, t)\| \leq M e^{\omega t} \text { for all } t \geq 0 .
$$

Example 2.3. Let $\Theta$ be a metric space and $\sigma$ a flow on $\Theta$. Consider also $\mathbf{T}=\{T(t)\}_{t \geq 0}$ a $C_{0}$-semigroup on the Banach space $X$. Then the pair $\pi_{T}=\left(\Phi_{T}, \sigma\right)$, where

$$
\Phi_{T}(\theta, t)=T(t), \quad \text { for all }(\theta, t) \in \Theta \times \mathbb{R}_{+},
$$

is a linear skew-product semiflow on $\mathcal{E}=X \times \Theta$.

Example 2.4. Let $X$ be a Banach space, $\Theta$ a locally compact metric space, $\mathbf{T}=\{T(t)\}_{t \geq 0}$ a $C_{0}$-semigroup and $\{U(\theta)\}_{\theta \in \Theta}$ a bounded strongly continuous family of idempotent operators with the property that

$$
U(\theta) T(t)=T(t) U(\theta), \quad \text { for all } t \geq 0, \theta \in \Theta,
$$

then the pair $\pi=(\Phi, \sigma)$ defined by

$$
\sigma(\theta, t)=\theta, \Phi(\theta, t)=U(\theta) T(t)
$$

is a strongly continuous skew-products semi-flow. 
Example 2.5. Let $\sigma$ be a flow on a compact metric space $\Theta$ and $\mathbf{T}=\{T(t)\}_{t \geq 0}$ a $C_{0}$-semigroup on $X$. For every strongly continuous function $D: \Theta \rightarrow B(X)$ there is a linear skew-product semiflow $\pi_{D}=$ $\left(\Phi_{D}, \sigma\right)$ on $\mathcal{E}=X \times \Theta$ such that

$\Phi_{D}(\theta, t) x=T(t) x+\int_{0}^{t} T(t-s) D(\theta \cdot t) \Phi_{D}(\theta, s) x d s$, for all $(x, \theta, t) \in \mathcal{E} \times \mathbb{R}_{+}$.

For the proof of this fact see [2].

Example 2.6. Let $\Theta=\mathbb{R}, \sigma(\theta, t)=\theta+t$ and consider $\mathcal{U}=$ $\{U(t, s)\}_{t \geq s \geq 0}$ an evolution family (process) on $X$. Define

$$
\Phi_{U}(\theta, t)=U(t+\theta, \theta), \quad \text { for all }(\theta, t) \in \mathbb{R} \times \mathbb{R}_{+} .
$$

Then the pair $\pi_{U}=\left(\Phi_{U}, \sigma\right)$ is a linear skew-product semiflow ( generated by the evolution family (process) $\mathcal{U}=\{U(t, s)\}_{t \geq s \geq 0}$ ). For the definition of an evolution family (process) see for instance [8, 17, 19, 20, 21].

Example 2.7. Let $\Theta$ be a compact metric space, $\sigma$ a semi-flow on $\Theta$, $X$ a Banach space $A: \Theta \rightarrow B(X)$ a continuous mapping. If $\Phi(\theta, t) x$ is the solution of the abstract Cauchy problem

$$
\left\{\begin{array}{l}
u^{\prime}(t)=A(\sigma(\theta, t)) u(t), \quad t \geq 0 \\
(0)=x
\end{array}\right.
$$

then the pair $\pi=(\Phi, \sigma)$ is a strongly continuous skew-product semiflow. Many of these equations are obtained when linearize the nonlinear differential equations (see for instance [25]).

Also, a large numbers of examples of strongly continuous skew-products semi-flow are provided in the recent literature (see for instance [14] ).

Definition 2.3. A strongly continuous skew-products semi-flow $\pi=$ $(\Phi, \sigma)$ is called uniformly exponentially stable (u.e.s) if there exists $N, \nu>0$ such that

$$
\|\Phi(\theta, t)\| \leq N e^{-\nu t}, \quad \text { for all } t \geq 0, \theta \in \Theta .
$$

In what follows for $f \in L^{1}\left(\mathbb{R}_{+}, X\right)$ we define $x_{f}: \Theta \times \mathbb{R}_{+} \rightarrow X$ the map given by

$$
x_{f}(\theta, t)=\int_{0}^{t} \Phi(\theta \cdot s, t-s) f(s) d s .
$$

From the Definition 2.2. it is easy to see that $x_{f}(\cdot, t) \in C_{b}(\Theta, X)$ for all $t \geq 0$. 
Definition 2.4. The Orlicz space $L^{\Psi}$ is said to be admissible to $\pi$ if for all $f \in L^{\Psi}(X)$ the map $u_{f}: \mathbb{R}_{+} \rightarrow C_{b}(\Theta, X)$ given by $u_{f}(t)=x_{f}(\cdot, t)$ belongs to $L^{\Psi}\left(C_{b}(\Theta, X)\right)$.

Remark 2.6. If we are in the case of the strongly continuous skewproduct semiflow provided by Example 2.4., then for all $\theta \in \Theta, x_{f}(\theta, \cdot)$ is the solution of the Cauchy problem

$$
\left\{\begin{array}{l}
u^{\prime}(t)=A(\sigma(\theta, t)) u(t)+f(t), \quad t \geq 0 \\
u(0)=0
\end{array}\right.
$$

\section{The main results}

Lemma 3.1. If the Orlicz space $L^{\Psi}$ is admissible to $\pi$ then there is $K>0$ such that

$$
\left\|u_{f}\right\|_{L^{\Psi}\left(C_{b}(\Theta, X)\right)} \leq K\|f\|_{L^{\Psi}(X)} .
$$

Proof. We set now $V: L^{\Psi}(X) \rightarrow L^{\Psi}\left(C_{b}(\Theta, X)\right), V f=u_{f}$. It is trivial to check that $V$ is a linear operator.

If we consider $\left\{f_{n}\right\}_{n \in \mathbb{N}} \subset L^{\Psi}(X), f \in L^{\Psi}(X)$, $g \in L^{\Psi}\left(C_{b}(\Theta, X)\right)$ such that

$$
f_{n} \stackrel{L^{\Psi}(X)}{\longrightarrow} f, \quad V f_{n} \stackrel{L^{\Psi}}{\stackrel{\left.C_{b}(\Theta, X)\right)}{\longrightarrow}} g
$$

then, by Remark 2.4, there exists a subsequence

$\left\{f_{n_{k}}\right\}_{k \in \mathbb{N}}$ of $\left\{f_{n}\right\}_{n \in \mathbb{N}}$ such that

$$
\begin{aligned}
f_{n_{k}} \rightarrow f \text { a.e. for } k \rightarrow \infty & , \quad V f_{n_{k}} \stackrel{L^{\Psi}}{\stackrel{\left.C_{b}(\Theta, X)\right)}{\longrightarrow}} g \text { a.e. for } k \rightarrow \infty \\
\left\|x_{f_{n_{k}}}(\theta, t)-x_{f}(\theta, t)\right\| & \leq \int_{0}^{t}\left\|\Phi(\theta \cdot s, t-s)\left(f_{n_{k}}(s)-f(s)\right)\right\| d s \\
& \leq M t e^{\omega t} \int_{0}^{t}\left\|f_{n_{k}}(s)-f(s)\right\| d s \\
& \leq M t e^{\omega t} a_{\Psi}(t)\left\|f_{n_{k}}-f\right\|_{L^{\Psi}(X)},
\end{aligned}
$$

for all $k \in \mathbb{N}, \theta \in \Theta, t \geq 0$, which implies that

$$
\left(V f_{n_{k}}\right)(t) \stackrel{C_{b}(\Theta, X)}{\longrightarrow}(V f)(t) \text { for all } t \geq 0
$$

It follows, using again the Remark 2.4, that $V f=g$, and hence $V$ is closed and so, by the Closed-Graph Theorem it is also bounded. So we 
obtain that

$$
\begin{aligned}
\left\|u_{f}\right\|_{L^{\Psi}\left(C_{b}(\Theta, X)\right)} & =\|V f\|_{L^{\Psi}\left(C_{b}(\Theta, X)\right)} \\
& \leq\|V\|\|f\|_{L^{\Psi}(X)}, \text { for all } f \in L^{\Psi}(X) \text { as required. }
\end{aligned}
$$

Lemma 3.2. If $L^{\Psi}$ is a Orlicz space, $h \in L^{\Psi}, h \geq 0$ and two constants $a, b>0$ such that $h(r) \leq a h(t)+b$, for all $r \geq t \geq 0$ with $r-t \leq 1$ then $h \in L^{\infty}$.

Proof. By the hypothesis we have that

$$
h(n+1) \leq a h(s)+b, \text { for all } n \in \mathbb{N} \text { and all } s \in[n, n+1]
$$

and from here

$$
h(n+1) \leq a \int_{n}^{n+1} h(s) d s+b \leq a a_{\Psi}(1)\|h\|_{L^{\Psi}}+b, \text { for all } n \in \mathbb{N}
$$

which implies that

$$
c=\sup _{n \in \mathbb{N}} h(n)<\infty .
$$

Using again the hypothesis, we obtain that

$$
h(t) \leq a h(n)+b \leq a c+b, \text { for all } n \in \mathbb{N} \text {, and all } t \in[n, n+1] .
$$

Lemma 3.3. If an Orlicz space $L^{\Psi}$ is admissible to $\pi$ then the following statements hold:

(i) For all $f \in L^{\Psi}(X)$ there exist $a, b>0$ such that

$$
\left\|\left|u _ { f } ( r ) \left\|\left|\leq a\left\|\left|u_{f}(t) \|\right|+b, \text { for all } r \geq t \geq 0 \text { with } r-t \leq 1 ;\right.\right.\right.\right.\right.
$$

(ii) For all $f \in L^{\Psi}(X)$ the map $u_{f}: \mathbb{R}_{+} \rightarrow C_{b}(\Theta, X)$ given by $u_{f}(t)=$ $x_{f}(\cdot, t)$ belongs also to $L^{\infty}\left(\mathbb{R}_{+}, C_{b}(\Theta, X)\right)$.

Proof. (i) We have that

$$
\begin{aligned}
x_{f}(\theta, r)= & \int_{0}^{r} \Phi(\theta \cdot s, r-s) f(s) d s \\
= & \int_{0}^{t} \Phi((\theta \cdot s) \cdot(t-s), r-t) \Phi(\theta \cdot s, t-s) f(s) d s \\
& +\int_{t}^{r} \Phi(\theta \cdot s, r-s) f(s) d s
\end{aligned}
$$




$$
=\Phi(\theta \cdot t, r-t) x_{f}(\theta, t)+\int_{t}^{r} \Phi(\theta \cdot s, r-s) f(s) d s,
$$

for all $\theta \in \Theta, \quad r \geq t \geq 0$.

It results that

$$
\begin{aligned}
\left\|x_{f}(\theta, r)\right\| & \leq M e^{\omega(r-t)}\left\|\left|u_{f}(t)\left\|\mid+\int_{t}^{r} M e^{\omega(r-s)}\right\| f(s) \| d s\right.\right. \\
& \leq M e^{\omega}\left\|\left|u_{f}(t)\left\|\mid+M e^{\omega} \int_{t}^{t+1}\right\| f(s) \| d s\right.\right. \\
& \leq M e^{\omega}\left\|\mid u_{f}(t)\right\|+M e^{\omega} a_{\Psi}(1)\|f\|_{L^{\Psi}(X)}
\end{aligned}
$$

for all $\theta \in \Theta, r \geq t \geq 0$ with $r-t \leq 1$.

The condition (ii) follows directly from (i) and Lemma 3.2.

Lemma 3.4. If $h_{1}, h_{2}: \mathbb{R}_{+} \rightarrow \mathbb{R}_{+}$satisfy the following conditions:

(i) $h_{1}(t) \leq h_{1}(s) h_{2}(t-s)$ for all $t \geq s \geq 0$;

(ii) $\sup _{t \in[0, a]} h_{2}(s)<\infty$, for all $a>0$;

(iii) $\inf _{t \geq 0} h_{2}(t)<1$, then there exist two constants $N, \nu>0$ such that

$$
h_{1}(t) \leq N e^{-\nu(t-s)} h_{1}(s) \text { for all } t \geq s \geq 0 .
$$

Proof. By (iii) there exist $\delta>0, \alpha \in(0,1)$ such that $h_{2}(\delta)<\alpha$. Let $t \geq s \geq 0$ and $n=\left[\frac{t-s}{\delta}\right]$, the largest integer less or equal than $\frac{t-s}{\delta}$. Then we have that

$$
\begin{aligned}
h_{1}(t) & \leq h_{2}(t-s-n \delta) h_{1}(n \delta+s) \\
& \leq \sup _{v \in[0, \delta]} h_{2}(v) h_{1}(n \delta+s) \\
& \leq \sup _{v \in[0, \delta]} h_{2}(v) h_{1}^{n}(\delta) h_{1}(s) \\
& \leq \sup _{v \in[0, \delta]} h_{2}(v) \alpha^{\frac{t-s}{\delta}-1} h_{1}(s) \\
& =N e^{-\nu(t-s)} h_{1}(s) \text { where } N=\frac{\sup _{v \in[0, \delta]} h_{2}(v)}{\alpha}, \nu=-\frac{\ln \alpha}{\delta},
\end{aligned}
$$

as required.

Theorem 3.1. If there exists an Orlicz space $L^{\Psi}$ admissible to $\pi$, then $\pi$ is u.e.s. 
Proof. First observe that if $L^{\Psi}$ is admissible to $\pi$ then by Lemma 3.3 we have that for all $f \in L^{\Psi}(X)$ the map $u_{f}: \mathbb{R}_{+} \rightarrow C_{b}(\Theta, X)$ given by $u_{f}(t)=x_{f}(\cdot, t)$ belongs also to $L^{\infty}\left(\mathbb{R}_{+}, C_{b}(\Theta, X)\right)$..

Let $x \in X, \theta_{0} \in \Theta$ and $f: \mathbb{R}_{+} \rightarrow X$,

$$
f(t)= \begin{cases}\Phi\left(\theta_{0}, t\right) x, & t \in[0,1] \\ 0, & t>1\end{cases}
$$

It is easy to check that $f \in L^{\Psi}(X)$ and $\|f\|_{L^{\Psi}(X)} \leq M e^{\omega} \frac{1}{\Psi^{-1}(1)}\|x\|$ and

$$
x_{f}\left(\theta_{0}, t\right)=\int_{0}^{1} \Phi\left(\theta_{0} \cdot s, t-s\right) \Phi\left(\theta_{0}, s\right) x d s=\Phi\left(\theta_{0}, t\right) x,
$$

for all $t \geq 1$ which implies that

$$
\begin{aligned}
\left\|\Phi\left(\theta_{0}, t\right) x\right\| & =\left\|x_{f}\left(\theta_{0}, t\right)\right\| \leq\left\|u_{f}(t)\right\| \| \\
& \leq\left\|u_{f}\right\|_{L^{\infty}\left(C_{b}(\Theta, X)\right)} \\
& \leq K\|f\|_{L^{\Psi}(X)} \\
& \leq K M e^{\omega} \frac{1}{\Phi^{-1}(1)}\|x\|
\end{aligned}
$$

for all $t \geq 1, \theta_{0} \in \Theta$ and all $x \in X$.

Hence there exits $L=M e^{\omega} \max \left\{\frac{K}{\Psi^{-1}(1)}, 1\right\}$ such that

$$
\|\Phi(\theta, t)\| \leq L, \quad \text { and } \text { for all } t \geq 0, \theta \in \Theta .
$$

Let us consider again $\theta_{0} \in \Theta, \delta>0, x \in X$ and $g: \mathbb{R}_{+} \rightarrow X$

$$
g(t)= \begin{cases}\Phi\left(\theta_{0}, t\right) x, & t \in[0, \delta] \\ 0, & t \geq \delta\end{cases}
$$

Then $g \in L^{\Psi}(X),\|g\|_{L^{\Psi}(X)} \leq \frac{L}{\Phi^{-1}\left(\frac{1}{\delta}\right)}\|x\|$.

It follows that

$$
\begin{aligned}
x_{g}\left(\theta_{0}, t\right) & =\int_{0}^{t} \Phi\left(\theta_{0} \cdot s, t-s\right) g(s) d s \\
& =\int_{0}^{t} \Phi\left(\theta_{0} \cdot s, t-s\right) \Phi\left(\theta_{0}, s\right) x d s \\
& =t \Phi\left(\theta_{0}, t\right) x,
\end{aligned}
$$


for all $t \in[0, \delta]$ and so

$$
\begin{aligned}
\frac{\delta^{2}}{2}\left\|\Phi\left(\theta_{0}, \delta\right) x\right\| & =\int_{0}^{\delta} s\left\|\Phi\left(\theta_{0}, \delta\right) x\right\| d s \\
& \leq \int_{0}^{\delta} s\left\|\Phi\left(\theta_{0} \cdot s, \delta-s\right) \Phi\left(\theta_{0}, s\right) x\right\| d s \\
& \leq L \int_{0}^{\delta}\left\|x_{g}\left(\theta_{0}, s\right)\right\| d s \\
& \leq L \int_{0}^{\delta}\left\|u_{g}(s)\right\| \mid d s \leq L a_{\Psi}(\delta)\left\|u_{g}\right\|_{L^{\Psi}\left(C_{b}(\Theta, X)\right)} \\
& \leq K L a_{\Psi}(\delta)\|g\|_{L^{\Psi}(X)} \\
& \leq K L^{2} a_{\Psi}(\delta) \frac{1}{\Psi^{-1}\left(\frac{1}{\delta}\right)}\|x\| \\
& \leq K L^{2} \delta\|x\|,
\end{aligned}
$$

for all $\theta_{0} \in \Theta, \delta>0, x \in X$.

Applying Lemma 3.4. to the functions $h_{1}, h_{2}: \mathbb{R}_{+} \rightarrow \mathbb{R}_{+}$defined by

$$
h_{1}(t)=\sup _{\theta \in \Theta}\|\Phi(\theta, t)\|, h_{2}(t)=\frac{2 K L^{2}}{t}
$$

it results that $\pi$ is u.e.s.

Remark. 3.1. It follows easily from Definition 2.3 that if $\pi$ is u.e.s then for all $f \in L^{\infty}\left(\mathbb{R}_{+}, X\right)$ the map $u_{f}: \mathbb{R}_{+} \rightarrow C_{b}(\Theta, X)$ given by $u_{f}(t)=x_{f}(\cdot, t)$ belongs also to $L^{\infty}\left(\mathbb{R}_{+}, C_{b}(\Theta, X)\right)$.

\section{References}

[1] S. N. Chow and H. Leiva, Dynamical spectrum for time-dependent linear systems in Banach spaces, Japan J. Indust. Appl. Math., 11 (1994), 379415.

[2] S. N. Chow and H. Leiva, Existence and roughness of the exponential dichotomy for linear skew-product semiflow in Banach space, J. Differential Equations, 102 (1995), 429-477.

[3] S. N. Chow and H. Leiva, Two definitions of exponential dichotomy for skew-product semiflow in Banach spaces, Proc. Amer. Math. Soc., 124(4) (1996), 429-477. 
[4] S. N. Chow and H. Leiva, Dynamical spectrum for skew-product flow in Banach spaces, Boundary Problems for Functional Differential Equations, World Sci. Publ., Singapore, 1995, 85-105.

[5] S. N. Chow and H. Leiva, Unbounded Perturbation of the Exponential Dichotomy for Evolution Equations, J. Differential Equations, 129 (1996), 509-531.

[6] S. Clark, Y. Latushkin, S. Montgomery-Smith and T. Randolph, Stability radius an internal versus external stability in Banach spaces: an evolution semigroup approach, SIAM J. Control Optimization, 38 (2000), 1757-1793.

[7] J. L Daleckij and M. G Krein, Stability of Differential Equations in Banach Space, Amer. Math. Scc, Providence, R.I. 1974.

[8] R. Datko, Uniform asymptotic stability of evolutionary processes in a Banach space, SIAM. J. Math. Analysis, 3 (1973), 428-445.

[9] J. K. Hale and S. M. Verduyn Lunel, Introduction to Functional Differential Equations, Appl. Math. Sci., 99, Springer-Verlag, New York, (1993).

[10] J. K. Hale, Asymptotic Behavior of Dissipative Systems, Mathematical Surveys and Monographs: Vol. 25, Providence (Rhode Island): Amer. Math. Soc., (1988).

[11] D. Henry, Geometric Theory of Nonlinear Parabolic Equations, Lect. Notes Math., 840, Springer-Verlag, Berlin, 1981.

[12] Y. Latushkin and A. M. Stepin, Weighted shift operators, spectral theory of linear extensions, and the multiplicative ergodic theory, Math. USSR Sbornik, 70 (1991), 143-163.

[13] Y. Latushkin and A. M. Stepin, Weighted translations operators and linear extensions of dynamical systems, Russian Math. Surveys, 46 (1991), 95-165.

[14] Y. Latushkin, S. Montgomery-Smith and T. Randolph, Evolutionary semigroups and dichotomy of linear skew-product flows on locally compact spaces with Banach fibers, J. Differential. Equations, 125 (1996), $73-116$.

[15] Y. Latushkin and R. Schnaubelt, Evolution semigroups, translation algebra and exponential dichotomy of cocycles, J. Differential. Equations, 159 (1999), 321-369.

[16] J. L. Massera and J. J. Schäffer, Linear Differential Equations and Function Spaces, Academic Press, New York, 1966. 
[17] N. van Minh, F. Räbiger and R. Schnaubelt, Exponential stability, exponential expansiveness and exponential dichotomy of evolution equations on the half-line, Int. Eq. Op. Theory, 32 (1998), 332-353.

[18] O. Perron, Die stabilitätsfrage bei differentialgeighungen, Math. Z., 32 (1930), 703-728.

[19] P. Preda, A. Pogan and C. Preda, $\left(L^{p}, L^{q}\right)$-admissibility and exponential dichotomy of evolutionary processes on half-line, Int. Eq. Op. Theory, 49(3) (2004), 405-418.

[20] P. Preda, A. Pogan and C. Preda, Admissibility and exponential dichotomy of evolutionary processes on half-line, Rend. Sem. Mat. Univ. Pol. Torino, 61(4) (2003), 461-473.

[21] P. Preda, A. Pogan and C. Preda, On ( $a, b)$-Dichotomy of evolutionary processes on half-line, Glasgow Math. J., 46, 2004, 217-225.

[22] R. J. Sacker and G. R. Sell, Existence of dichotomies and invariant splitting for linear differential Systems I, II, III, J. Differential. Equations, 15 (1974), 429-458; 22 (1976), 478-522.

[23] R. J. Sacker and G. R. Sell, A spectral theory for linear differential systems, J. Differential. Equations, 27 (1978), 330-358.

[24] R. J. Sacker and G. R. Sell, The spectrum of invariant manifold, J. Differential. Equations, 38 (1980), 135-160.

[25] R. J. Sacker and G. R. Sell, Dichotomies for linear evolutionary equations in Banach spaces, J. Differential. Equations, 113 (1994), 1767.

[26] J. Selgrade, Isolated invariant sets for flows on vector bundles, Trans. Amer. Math. Soc., 203 (1975), 359-390.

[27] A. C. Zaanen, Integration, North-Holland, Amsterdam, 1967.

Department of Electrical Engineering

University of California

Los Angeles, CA 90095

U.S.A.

(E-mail : preda@ee.ucla.edu) 


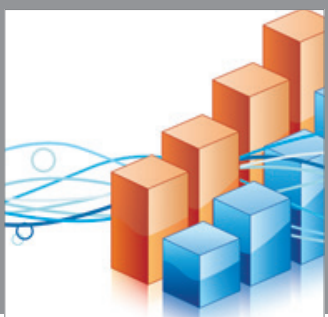

Advances in

Operations Research

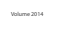

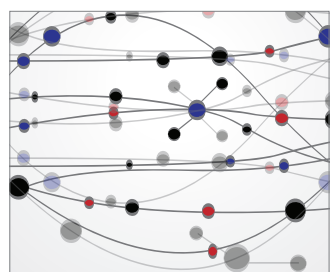

\section{The Scientific} World Journal
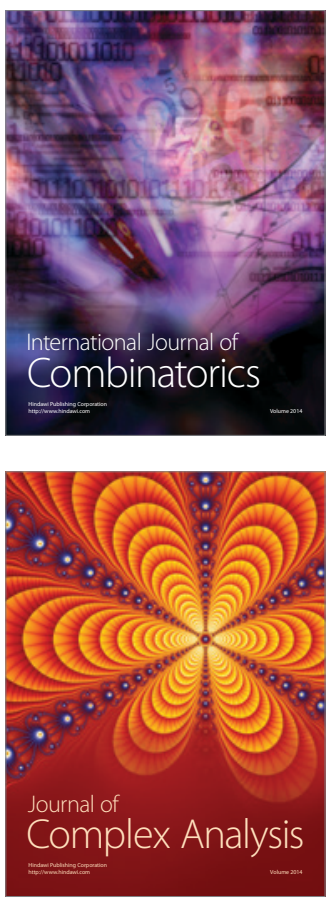

International Journal of

Mathematics and

Mathematical

Sciences
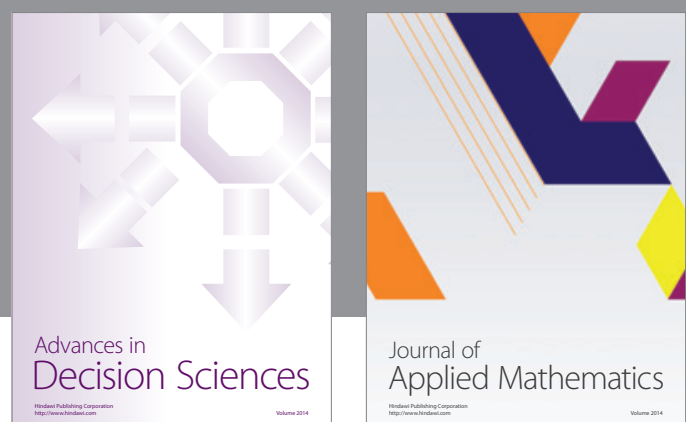

Journal of

Applied Mathematics
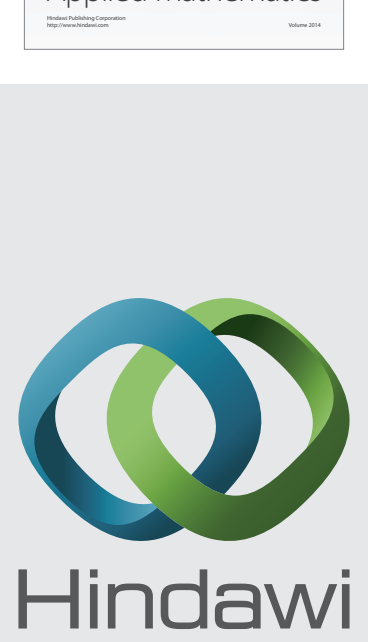

Submit your manuscripts at http://www.hindawi.com
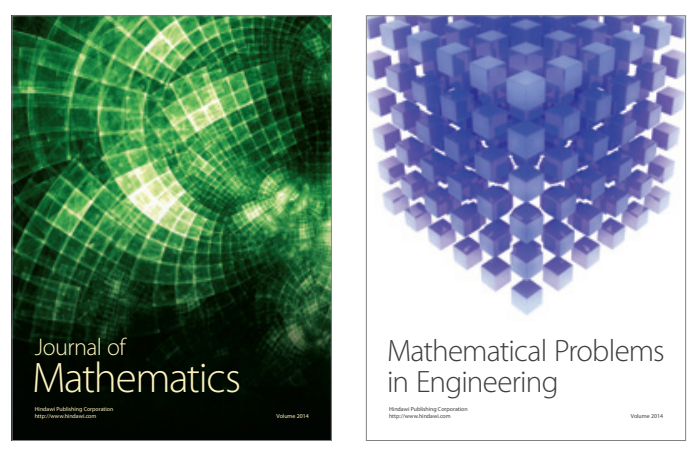

Mathematical Problems in Engineering
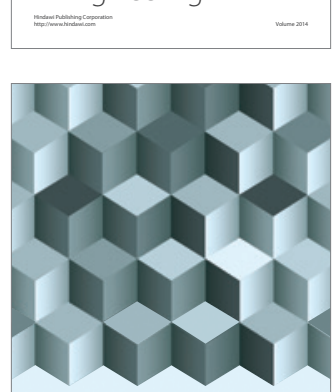

Journal of

Function Spaces
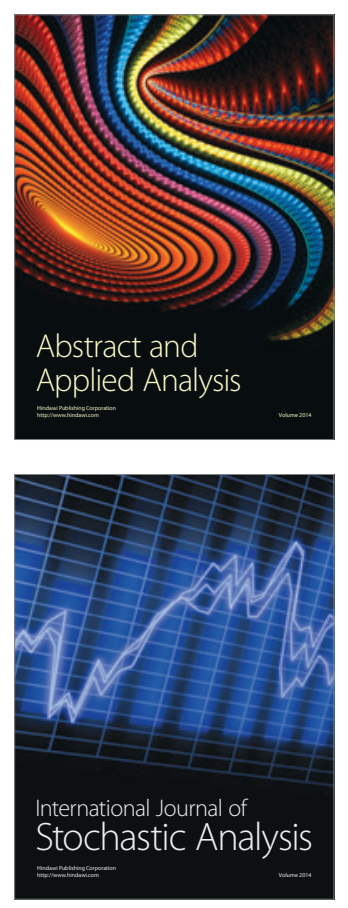

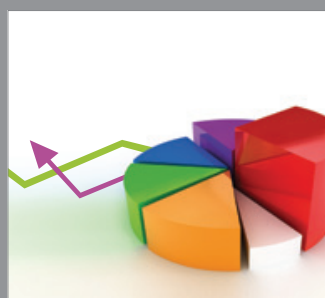

ournal of

Probability and Statistics

Promensencen
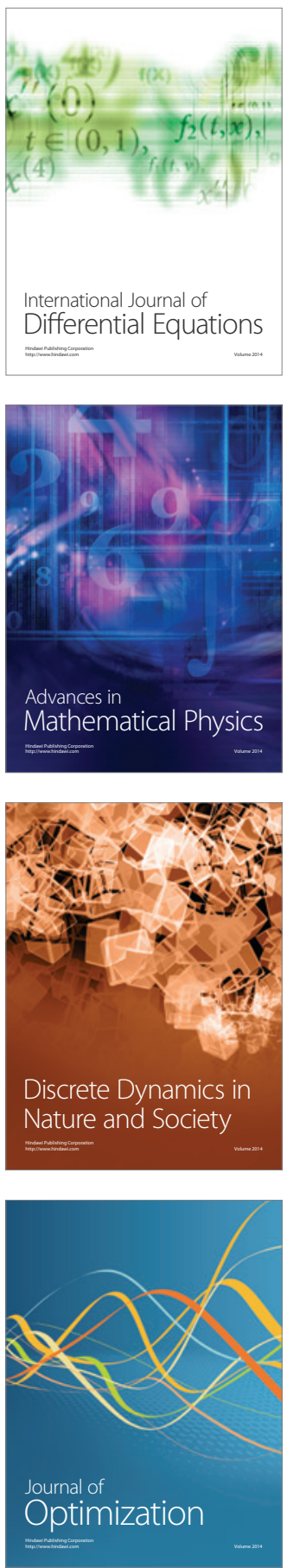